ANNALES

POLONICI MATHEMATICI

$98.1(2010)$

\title{
Long time behaviour of a Cahn-Hilliard system coupled with viscoelasticity
}

\author{
by IrenA PAWŁow and WojCIECH M. ZAJĄCZKOWski (Warszawa)
}

\begin{abstract}
The long-time behaviour of a unique regular solution to the Cahn-Hilliard system coupled with viscoelasticity is studied. The system arises as a model of the phase separation process in a binary deformable alloy. It is proved that for a sufficiently regular initial data the trajectory of the solution converges to the $\omega$-limit set of these data. Moreover, it is shown that every element of the $\omega$-limit set is a solution of the corresponding stationary problem.
\end{abstract}

1. Introduction. As a continuation of [5], we study the asymptotic behaviour as $t \rightarrow \infty$ of a regular solution to the following Cahn-Hilliard system coupled with viscoelasticity:

$$
\begin{array}{ll}
\boldsymbol{u}_{t t}-\nabla \cdot\left[W_{, \varepsilon}(\varepsilon(\boldsymbol{u}), \chi)+\nu \boldsymbol{A} \varepsilon\left(\boldsymbol{u}_{t}\right)\right]=\boldsymbol{b} & \text { in } \Omega^{\infty}=\Omega \times(0, \infty), \\
\boldsymbol{u}(0)=\boldsymbol{u}_{0}, \quad \boldsymbol{u}_{t}(0)=\boldsymbol{u}_{1} & \text { in } \Omega, \\
\boldsymbol{u}=\mathbf{0} & \text { on } S^{\infty}=S \times(0, \infty),
\end{array}
$$

$$
\begin{array}{cl}
\chi_{t}-\Delta \mu=0 & \text { in } \Omega^{\infty}, \\
\chi(0)=\chi_{0} & \text { in } \Omega, \\
\boldsymbol{n} \cdot \nabla \mu=0 & \text { on } S^{\infty}, \\
\mu=-\gamma \Delta \chi+\psi^{\prime}(\chi)+W_{, \chi}(\boldsymbol{\varepsilon}(\boldsymbol{u}), \chi) & \text { in } \Omega^{\infty}, \\
\boldsymbol{n} \cdot \nabla \chi=0 & \text { on } S^{\infty},
\end{array}
$$

where $\Omega \subset \mathbb{R}^{3}$ is a bounded domain with a smooth boundary $S$; the unknowns are the fields $\boldsymbol{u}: \Omega^{\infty} \rightarrow \mathbb{R}^{3}, \chi: \Omega^{\infty} \rightarrow \mathbb{R}$, and $\mu: \Omega^{\infty} \rightarrow \mathbb{R}$, representing respectively the displacement vector, the order parameter and the chemical potential; $\boldsymbol{\varepsilon}(\boldsymbol{u})=\frac{1}{2}\left(\nabla \boldsymbol{u}+(\nabla \boldsymbol{u})^{T}\right)$ is the linearized strain tensor; the functions $W(\varepsilon(\boldsymbol{u}), \chi)$ and $\psi(\chi)$ are specified below, and $\nu, \gamma$ are positive constants.

2010 Mathematics Subject Classification: 35K50, 35K60, 35L20, 35Q72.

Key words and phrases: Cahn-Hilliard, viscoelasticity system, phase separation, long-time behaviour. 
The system arises as a model, regularized by a viscous damping, of the phase separation process in a deformable two-component $a-b$ alloy cooled below a critical temperature. In the previous paper [5] we have proved the existence and uniqueness of a global in time, regular solution to this system. Moreover, we have shown the existence of an absorbing set. Our objective in the present paper is to study the asymptotic behaviour of the solution as $t \rightarrow \infty$.

System (1.1)-(1.3) represents balance laws of linear momentum, mass, and the equation for the chemical potential. The associated free energy density has the Landau-Ginzburg form

$$
f(\varepsilon(\boldsymbol{u}), \chi, \nabla \chi)=W(\varepsilon(\boldsymbol{u}), \chi)+\psi(\chi)+\frac{\gamma}{2}|\nabla \chi|^{2},
$$

where

$$
W(\varepsilon(\boldsymbol{u}), \chi)=\frac{1}{2}(\varepsilon(\boldsymbol{u})-\bar{\varepsilon}(\chi)) \cdot \boldsymbol{A}(\varepsilon(\boldsymbol{u})-\bar{\varepsilon}(\chi)),
$$

and

$$
\psi=\frac{1}{4}\left(1-\chi^{2}\right)^{2}
$$

represent respectively the elastic energy and the double-well potential; the positive constant $\gamma$ is related to the surface tension.

The order parameter $\chi$ characterizes the material phase. In case of a binary alloy it is related to the volumetric fraction of one of the two phases, characterized by different crystalline structures of the components. We shall assume that $\chi=-1$ is identified with phase $a$ and $\chi=1$ with phase $b$.

The elasticity tensor $\boldsymbol{A}=\left(A_{i j k l}\right)$ and the eigenstrain tensor $\bar{\varepsilon}(\chi)=$ $\left(\bar{\varepsilon}_{i j}(\chi)\right)$ are given by

$$
\boldsymbol{A} \boldsymbol{\varepsilon}(\boldsymbol{u})=\bar{\lambda} \operatorname{tr} \boldsymbol{\varepsilon}(\boldsymbol{u}) \boldsymbol{I}+2 \bar{\mu} \boldsymbol{\varepsilon}(\boldsymbol{u}), \quad \overline{\boldsymbol{\varepsilon}}(\chi)=(1-z(\chi)) \bar{\varepsilon}_{a}+z(\chi) \overline{\boldsymbol{\varepsilon}}_{b},
$$

where $\boldsymbol{I}$ is the identity tensor, $\bar{\lambda}, \bar{\mu}$ are the Lamé constants satisfying $\bar{\mu}>0$, $3 \bar{\lambda}+2 \bar{\mu}>0, \bar{\varepsilon}_{a}, \bar{\varepsilon}_{b}$ are the constant eigenstrains of phases $a, b$, and $z: \mathbb{R} \rightarrow$ $[0,1]$ is a sufficiently smooth interpolation function such that

$$
z(\chi)=0 \quad \text { for } \chi \leq-1 \text { and } z(\chi)=1 \text { for } \chi \geq 1 .
$$

The term $\nu \boldsymbol{A} \boldsymbol{\varepsilon}\left(\boldsymbol{u}_{t}\right)$, with $\nu=$ const $>0$, represents the viscous stress tensor; $\nu$ is the viscosity coefficient. The derivatives of $W(\varepsilon(\boldsymbol{u}), \chi)$ with respect to $\varepsilon$ and $\chi$, given by

$$
\begin{aligned}
W_{, \varepsilon}(\varepsilon(\boldsymbol{u}), \chi) & =\boldsymbol{A}(\varepsilon(\boldsymbol{u})-\bar{\varepsilon}(\chi)), \\
W_{, \chi}(\varepsilon(\boldsymbol{u}), \chi) & =-\overline{\boldsymbol{\varepsilon}}^{\prime}(\chi) \cdot \boldsymbol{A}(\varepsilon(\boldsymbol{u})-\overline{\boldsymbol{\varepsilon}}(\chi)),
\end{aligned}
$$

denote respectively the elastic stress tensor and the elastic contribution to the chemical potential. For a detailed description of system (1.1)-(1.3) and a discussion of related literature we refer to [5]. 
By introducing the linear elasticity operator

$$
\boldsymbol{u} \mapsto \boldsymbol{Q u}=\nabla \cdot(\boldsymbol{A} \boldsymbol{\varepsilon}(\boldsymbol{u}))=\bar{\mu} \Delta \boldsymbol{u}+(\bar{\lambda}+\bar{\mu}) \nabla(\nabla \cdot \boldsymbol{u})
$$

with the domain $D(\boldsymbol{Q})=\boldsymbol{H}^{2}(\Omega) \cap \boldsymbol{H}_{0}^{1}(\Omega)$, and the auxiliary constant quantities

$$
\boldsymbol{B}=-\boldsymbol{A}\left(\bar{\varepsilon}_{b}-\bar{\varepsilon}_{a}\right), \quad D=-\boldsymbol{B} \cdot\left(\bar{\varepsilon}_{b}-\bar{\varepsilon}_{a}\right), \quad E=-\boldsymbol{B} \cdot \bar{\varepsilon}_{a}
$$

we have

$$
\begin{aligned}
& W_{, \varepsilon}(\varepsilon(\boldsymbol{u}), \chi)=\boldsymbol{A} \boldsymbol{\varepsilon}(\boldsymbol{u})-\boldsymbol{A} \bar{\varepsilon}_{a}+z(\chi) \boldsymbol{B}, \\
& W_{, \chi}(\varepsilon(\boldsymbol{u}), \chi)=z^{\prime}(\chi)(\boldsymbol{B} \cdot \boldsymbol{\varepsilon}(\boldsymbol{u})+D z(\chi)+E), \\
& \nabla \cdot W_{, \varepsilon}(\boldsymbol{\varepsilon}(\boldsymbol{u}), \chi)=\boldsymbol{Q u}+z^{\prime}(\chi) \boldsymbol{B} \nabla \chi .
\end{aligned}
$$

On account of (1.9)-(1.11) it is convenient to recast system (1.1)-(1.3) into the following simplified form:

$$
\begin{aligned}
& \boldsymbol{u}_{t t}-\boldsymbol{Q u}-\nu \boldsymbol{Q} \boldsymbol{u}_{t}=z^{\prime}(\chi) \boldsymbol{B} \nabla \chi+\boldsymbol{b} \quad \text { in } \Omega^{\infty}, \\
& \boldsymbol{u}(0)=\boldsymbol{u}_{0}, \quad \boldsymbol{u}_{t}(0)=\boldsymbol{u}_{1} \quad \text { in } \Omega \text {, } \\
& \boldsymbol{u}=\mathbf{0} \\
& \text { on } S^{\infty} \text {, } \\
& \chi_{t}-\Delta \mu=0 \quad \text { in } \Omega^{\infty} \text {, } \\
& \chi(0)=\chi_{0} \quad \text { in } \Omega \text {, } \\
& \boldsymbol{n} \cdot \nabla \mu=0 \quad \text { on } S^{\infty} \text {, } \\
& \begin{array}{ll}
\mu=-\gamma \Delta \chi+\psi^{\prime}(\chi)+z^{\prime}(\chi)(\boldsymbol{B} \cdot \boldsymbol{\varepsilon}(\boldsymbol{u})+D z(\chi)+E) & \text { in } \Omega^{\infty}, \\
\boldsymbol{n} \cdot \nabla \chi=0 & \text { on } S^{\infty} .
\end{array}
\end{aligned}
$$

It has been proved in [5] (see Theorem 2.1 below) that system (1.1)-(1.3) admits a unique global solution $(\boldsymbol{u}, \chi, \mu)$ such that

$$
\begin{aligned}
& \boldsymbol{u} \in C^{1}\left([0, \infty) ; \boldsymbol{H}^{2}(\Omega) \cap \boldsymbol{H}_{0}^{1}(\Omega)\right) \cap C^{2}\left([0, \infty) ; \boldsymbol{H}_{0}^{1}(\Omega)\right), \\
& \chi \in C\left([0, \infty) ; H_{N}^{2}(\Omega)\right) \cap C^{1}\left([0, \infty) ; L_{2}(\Omega)\right), \\
& \mu \in C\left([0, \infty) ; H_{N}^{2}(\Omega)\right), \quad f_{\Omega} \chi(t) d x=\chi_{m}:=f_{\Omega} \chi_{0} d x \quad \text { for all } t \in[0, \infty),
\end{aligned}
$$

for initial data satisfying

$$
\begin{aligned}
& \left(\boldsymbol{u}(0), \boldsymbol{u}_{t}(0), \boldsymbol{u}_{t t}(0), \chi(0), \chi_{t}(0)\right) \in \mathcal{W} \\
& :=\left\{\left(\boldsymbol{H}^{2}(\Omega) \cap \boldsymbol{H}_{0}^{1}(\Omega)\right) \times\left(\boldsymbol{H}^{2}(\Omega) \cap \boldsymbol{H}_{0}^{1}(\Omega)\right) \times \boldsymbol{H}_{0}^{1}(\Omega) \times H_{N}^{2}(\Omega) \times L_{2}(\Omega)\right\},
\end{aligned}
$$

where

$$
H_{N}^{2}(\Omega)=\left\{\xi: \xi \in H^{2}(\Omega), \boldsymbol{n} \cdot \nabla \xi=0 \text { on } S\right\} .
$$

Thus, the solution defines a nonlinear, strongly continuous semigroup

$$
\begin{aligned}
& S(t): \mathcal{W} \ni\left(\boldsymbol{u}(0), \boldsymbol{u}_{t}(0), \boldsymbol{u}_{t t}(0), \chi(0), \chi_{t}(0)\right) \mapsto \\
&\left(\boldsymbol{u}(t), \boldsymbol{u}_{t}(t), \boldsymbol{u}_{t t}(t), \chi(t), \chi_{t}(t)\right) \in \mathcal{W}, \quad t \in[0, \infty) .
\end{aligned}
$$


In this paper we prove that for any initial data belonging to $\mathcal{W}$ the trajectory of the solution converges as $t \rightarrow \infty$ to the $\omega$-limit set of these data.

Moreover, we show that the $\omega$-limit set is a compact and connected subset of the space

$$
\mathcal{Z}:=\boldsymbol{H}_{0}^{1}(\Omega) \times \boldsymbol{H}_{0}^{1}(\Omega) \times \boldsymbol{L}_{2}(\Omega) \times H^{1}(\Omega) \times\left(H^{1}(\Omega)\right)^{\prime},
$$

and enjoys the standard properties, namely it is positive invariant with respect to semigroup $S(t)$ defined by the solution, and the total energy functional is constant on this set. We also prove that every element of the $\omega$-limit set is a solution of the corresponding stationary problem.

In the proof of these results we use arguments similar to those applied in [7] for the single Cahn-Hilliard equation, and the procedure of long-time analysis devised in [1], 2] for phase-field models.

We use the same notation as in 5]. Vectors and tensors are denoted by bold letters. A dot designates the inner product irrespective of the space in question, e.g. for vectors $\boldsymbol{a}=\left(a_{i}\right), \tilde{\boldsymbol{a}}=\left(\tilde{a}_{i}\right)$ and tensors $\boldsymbol{B}=\left(B_{i j}\right)$, $\tilde{\boldsymbol{B}}=\left(\tilde{B}_{i j}\right)$ we write $\boldsymbol{a} \cdot \tilde{\boldsymbol{a}}=a_{i} \tilde{a}_{i}, \boldsymbol{B} \cdot \tilde{\boldsymbol{B}}=B_{i j} \tilde{B}_{i j}$. Here and throughout the summation convention over repeated indices is used.

The symbols $\nabla$ and $\nabla \cdot$ denote the gradient and the divergence operators. For the divergence we use the convention of contraction over the last index, e.g. $\nabla \cdot \varepsilon=\left(\partial \varepsilon_{i j} / \partial x_{j}\right)$.

For simplicity, the space and time derivatives (material) are denoted by $f_{, i}=\partial f / \partial x_{i}, f_{t}=\partial f / \partial t$. Moreover, for $\varepsilon=\left(\varepsilon_{i j}\right)$ we write $W_{, \varepsilon}(\varepsilon, \chi)=$ $\left(\partial W(\varepsilon, \chi) / \partial \varepsilon_{i j}\right)$. We use the standard Sobolev space notation. In addition, the spaces of vector- or tensor-valued functions are indicated by bold letters.

2. Main results. First we recall the existence and uniqueness result for (1.1)-(1.3), proved in [5] under the following assumptions:

(A1) $\Omega \subset \mathbb{R}^{3}$ is a bounded domain with the boundary $S$ of class at least $C^{2}, T>0$ is an arbitrary fixed number.

(A2) The Lamé coefficients $\bar{\mu}, \bar{\lambda}$ satisfy

$$
\bar{\mu}>0, \quad 3 \bar{\lambda}+2 \bar{\mu}>0 .
$$

This ensures that the elasticity tensor $\boldsymbol{A}$ is coercive and bounded, i.e.,

$$
c_{*}|\varepsilon|^{2} \leq \varepsilon \cdot \boldsymbol{A} \varepsilon \leq c^{*}|\varepsilon|^{2}
$$

for all symmetric second order tensors $\varepsilon$ in $\mathbb{R}^{3}$, with positive constants $c_{*}$ and $c^{*}$. Moreover, due to this condition the operator $\boldsymbol{Q}$ given by (1.9) is strongly elliptic and satisfies

$$
\underline{c}_{Q}\|\boldsymbol{u}\|_{\boldsymbol{H}^{2}(\Omega)} \leq\|\boldsymbol{Q u}\|_{\boldsymbol{L}_{2}(\Omega)} \quad \text { for } \boldsymbol{u} \in D(\boldsymbol{Q})=\boldsymbol{H}^{2}(\Omega) \cap \boldsymbol{H}_{0}^{1}(\Omega)
$$

with a positive constant $\underline{c}_{Q}$. 
(A3) $W(\varepsilon(\boldsymbol{u}), \chi)$ is given by $(1.5)$; the function $z: \mathbb{R} \rightarrow[0,1]$ is of class $C^{2}$ satisfying (1.8) and

$$
\left|z^{\prime}(\chi)\right|+\left|z^{\prime \prime}(\chi)\right| \leq c \quad \text { for all } \chi \in \mathbb{R} .
$$

The auxiliary quantities $\boldsymbol{B}, D$ and $E$ are defined in (1.10).

(A4) $\psi(\chi)$ is given by (1.6).

(A5) $\gamma$ and $\nu$ are positive constants.

The next assumption concerns the initial data. In addition to

$$
\boldsymbol{u}(0)=\boldsymbol{u}_{0}, \quad \boldsymbol{u}_{t}(0)=\boldsymbol{u}_{1}, \quad \chi(0)=\chi_{0} \quad \text { in } \Omega,
$$

we introduce in compatibility with (1.12)-(1.14) the initial conditions corresponding to $\boldsymbol{u}_{t t}(0)$ and $\chi_{t}(0)$ :

$$
\begin{aligned}
\boldsymbol{u}_{2} & :=\boldsymbol{u}_{t t}(0)=\boldsymbol{Q} \boldsymbol{u}_{0}+\nu \boldsymbol{Q} \boldsymbol{u}_{1}+z^{\prime}\left(\chi_{0}\right) \boldsymbol{B} \nabla \chi_{0}+\boldsymbol{b}(0), \\
\chi_{1} & :=\chi_{t}(0)=\Delta \mu(0) \\
& =-\gamma \Delta^{2} \chi_{0}+\Delta\left[\psi^{\prime}\left(\chi_{0}\right)+z^{\prime}\left(\chi_{0}\right)\left(\boldsymbol{B} \cdot \boldsymbol{\varepsilon}\left(\boldsymbol{u}_{0}\right)+D z\left(\chi_{0}\right)+E\right)\right] \quad \text { in } \Omega .
\end{aligned}
$$

We assume

(A6) $\boldsymbol{u}_{0}, \boldsymbol{u}_{1} \in \boldsymbol{H}^{2}(\Omega) \cap \boldsymbol{H}_{0}^{1}(\Omega), \boldsymbol{u}_{2} \in \boldsymbol{H}_{0}^{1}(\Omega), \chi_{0} \in H_{N}^{2}(\Omega):=\left\{\xi \in H^{2}(\Omega)\right.$ : $\boldsymbol{n} \cdot \nabla \xi=0$ on $S\}, \chi_{m}:=f_{\Omega} \chi_{0} d x<\infty, \chi_{1} \in L_{2}(\Omega)$, which implies that $\boldsymbol{u}_{0} \in \boldsymbol{H}^{3}(\Omega) \cap \boldsymbol{H}_{0}^{1}(\Omega), \chi_{0} \in H^{4}(\Omega) \cap H_{N}^{2}(\Omega)$.

As regards the external force, we require

(A7) $\boldsymbol{b} \in L_{1}\left(0, \infty ; \boldsymbol{L}_{2}(\Omega)\right) \cap W_{\infty}^{1}\left(0, \infty ; \boldsymbol{H}_{0}^{1}(\Omega)\right)$.

The existence theorem is as follows:

Theorem 2.1 (see [5, Thms. 2.1, 2.3]). Let assumptions (A1)-(A7) hold true. Then problem (1.1)-(1.3) (in simplified formulation (1.12)-(1.14)) admits a unique global solution $(\boldsymbol{u}, \chi, \mu)$ on $[0, \infty)$ such that

$$
\begin{aligned}
& \boldsymbol{u} \in C^{1}\left([0, \infty) ; \boldsymbol{H}^{2}(\Omega) \cap \boldsymbol{H}_{0}^{1}(\Omega)\right) \cap C^{2}\left([0, \infty) ; \boldsymbol{H}_{0}^{1}(\Omega)\right), \\
& \chi \in C\left([0, \infty) ; H_{N}^{2}(\Omega)\right) \cap C^{1}\left([0, \infty) ; L_{2}(\Omega)\right), \\
& \mu \in C\left([0, \infty) ; H_{N}^{2}(\Omega)\right), \quad f_{\Omega} \chi(t) d x=\chi_{m} \quad \text { for all } t \in[0, \infty), \\
& \boldsymbol{u}_{t} \in L_{2}\left(0, \infty ; \boldsymbol{H}_{0}^{1}(\Omega)\right), \quad \nabla \mu \in L_{2}\left(0, \infty ; \boldsymbol{L}_{2}(\Omega)\right),
\end{aligned}
$$

$$
\boldsymbol{u}(0)=\boldsymbol{u}_{0}, \quad \boldsymbol{u}_{t}(0)=\boldsymbol{u}_{1}, \quad \boldsymbol{u}_{t t}(0)=\boldsymbol{u}_{2}, \quad \chi(0)=\chi_{0}, \quad \chi_{t}(0)=\chi_{1},
$$

and, for any $t \in[0, \infty)$ and any fixed number $T>0$,

$$
\begin{array}{ll}
\boldsymbol{u}_{t t} \in L_{2}\left(t, t+T ; \boldsymbol{H}^{2}(\Omega) \cap \boldsymbol{H}_{0}^{1}(\Omega)\right), & \boldsymbol{u}_{t t t} \in L_{2}\left(t, t+T ;\left(\boldsymbol{H}_{0}^{1}(\Omega)\right)^{\prime}\right), \\
\chi_{t} \in L_{2}\left(t, t+T ; H_{N}^{2}(\Omega)\right), & \chi_{t t} \in L_{2}\left(t, t+T ;\left(H_{N}^{2}(\Omega)\right)^{\prime}\right), \\
\mu \in L_{2}\left(t, t+T ; H^{1}(\Omega)\right), & \mu_{t} \in L_{2}\left(t, t+T ; L_{2}(\Omega)\right) .
\end{array}
$$

Furthermore, the solution satisfies the following estimates: 
- uniformly in time,

$$
\begin{gathered}
\|\boldsymbol{u}\|_{C\left([0, \infty) ; \boldsymbol{H}_{0}^{1}(\Omega)\right)}+\left\|\boldsymbol{u}_{t}\right\|_{C\left([0, \infty) ; \boldsymbol{L}_{2}(\Omega)\right)}+\|\chi\|_{C\left([0, \infty) ; H^{1}(\Omega)\right)} \\
+\left\|\boldsymbol{u}_{t}\right\|_{L_{2}\left(0, \infty ; \boldsymbol{H}_{0}^{1}(\Omega)\right)}+\|\nabla \mu\|_{L_{2}\left(0, \infty ; \boldsymbol{L}_{2}(\Omega)\right)} \leq c_{0}, \\
\|\boldsymbol{u}\|_{C^{1}\left([0, \infty) ; \boldsymbol{H}^{2}(\Omega)\right)}+\left\|\boldsymbol{u}_{t t}\right\|_{C\left([0, \infty) ; \boldsymbol{H}_{0}^{1}(\Omega)\right)}+\|\chi\|_{C\left([0, \infty) ; H_{N}^{2}(\Omega)\right)} \\
+\left\|\chi_{t}\right\|_{C\left([0, \infty) ; L_{2}(\Omega)\right)}+\|\mu\|_{C\left([0, \infty) ; H_{N}^{2}(\Omega)\right)} \leq c
\end{gathered}
$$

where

$$
\begin{gathered}
c_{0}=c_{0}\left(\left\|\boldsymbol{u}_{0}\right\|_{\boldsymbol{H}_{0}^{1}(\Omega)},\left\|\boldsymbol{u}_{1}\right\|_{\boldsymbol{L}_{2}(\Omega)},\left\|\chi_{0}\right\|_{H^{1}(\Omega)},\|\boldsymbol{b}\|_{L_{1}\left(0, \infty ; \boldsymbol{L}_{2}(\Omega)\right)}\right) \\
c=c\left(\left\|\boldsymbol{u}_{0}\right\|_{\boldsymbol{H}^{2}(\Omega)},\left\|\boldsymbol{u}_{1}\right\|_{\boldsymbol{H}^{2}(\Omega)},\left\|\boldsymbol{u}_{2}\right\|_{\boldsymbol{H}^{1}(\Omega)},\left\|\chi_{0}\right\|_{H_{N}^{2}(\Omega)},\left\|\chi_{1}\right\|_{L_{2}(\Omega)},\right. \\
\left.\|\boldsymbol{b}\|_{W_{\infty}^{1}\left(0, \infty ; \boldsymbol{L}_{2}(\Omega)\right)}\right)
\end{gathered}
$$

are positive constants;

- for any $t \in[0, \infty)$ and any fixed $T>0$,

$$
\begin{aligned}
& \|\chi\|_{L_{2}\left(t, t+T ; H_{N}^{2}(\Omega)\right)}+\|\mu\|_{L_{2}\left(t, t+T ; H^{1}(\Omega)\right)} \leq c\left(c_{0}\right)\left(T^{1 / 2}+1\right) \\
& \left\|\boldsymbol{u}_{t t}\right\|_{L_{2}\left(t, t+T ; \boldsymbol{H}^{2}(\Omega)\right)}+\left\|\chi_{t}\right\|_{L_{2}\left(t, t+T ; H_{N}^{2}(\Omega)\right)} \leq c\left(T^{1 / 2}+1\right) \\
& \left\|\boldsymbol{u}_{t t t}\right\|_{L_{2}\left(t, t+T ;\left(\boldsymbol{H}_{0}^{1}(\Omega)\right)^{\prime}\right)}+\left\|\chi_{t t}\right\|_{L_{2}\left(t, t+T ;\left(H_{N}^{2}(\Omega)\right)^{\prime}\right)} \\
& \quad+\left\|\mu_{t}\right\|_{L_{2}\left(t, t+T ; L_{2}(\Omega)\right)} \leq c\left(T^{1 / 2}+1\right)
\end{aligned}
$$

with constants $c_{0}, c$ as above.

Let us introduce the spaces

$$
\begin{aligned}
\mathcal{W}:= & \left(\boldsymbol{H}^{2}(\Omega) \cap \boldsymbol{H}_{0}^{1}(\Omega)\right) \times\left(\boldsymbol{H}^{2}(\Omega) \cap \boldsymbol{H}_{0}^{1}(\Omega)\right) \\
& \times \boldsymbol{H}_{0}^{1}(\Omega) \times H_{N}^{2}(\Omega) \times L_{2}(\Omega), \\
\mathcal{Z}:= & \boldsymbol{H}_{0}^{1}(\Omega) \times \boldsymbol{H}_{0}^{1}(\Omega) \times \boldsymbol{L}_{2}(\Omega) \times H^{1}(\Omega) \times\left(H^{1}(\Omega)\right)^{\prime} .
\end{aligned}
$$

In view of (2.7) the solution in Theorem 2.1 generates a strongly continuous, nonlinear semigroup

$$
S(t): \zeta_{0} \in \mathcal{W} \mapsto \zeta(t) \in \mathcal{W}, \quad t \geq 0,
$$

where

$$
\zeta_{0}:=\left(\boldsymbol{u}_{0}, \boldsymbol{u}_{1}, \boldsymbol{u}_{2}, \chi_{0}, \chi_{1}\right), \quad \zeta(t):=\left(\boldsymbol{u}(t), \boldsymbol{u}_{t}(t), \boldsymbol{u}_{t t}(t), \chi(t), \chi_{t}(t)\right) .
$$

Let us introduce the $\omega$-limit set of the initial data $\zeta_{0} \in \mathcal{W}$ :

$$
\begin{aligned}
\omega\left(\zeta_{0}\right):=\left\{\zeta_{\infty}=\left(\boldsymbol{u}_{\infty}, \boldsymbol{u}_{\infty, t},\right.\right. & \left.\boldsymbol{u}_{\infty, t t}, \chi_{\infty}, \chi_{\infty, t}\right) \in \mathcal{W} \subset \mathcal{Z}: \\
& \exists\left\{t_{n}\right\} \subset(0, \infty), t_{n} \rightarrow \infty \text { and } \\
& \left.\zeta\left(t_{n}\right)=S\left(t_{n}\right) \zeta_{0} \rightarrow \zeta_{\infty} \text { strongly in } \mathcal{Z}\right\} .
\end{aligned}
$$

The main result of this paper is stated in the following 
TheOrem 2.2. Assume that (A1)-(A7) hold. Let $S(t): \mathcal{W} \rightarrow \mathcal{W}, t \geq 0$, be the nonlinear semigroup generated by the unique solution of system (1.1)(1.3). Then

(i) The $\omega$-limit set $\omega\left(\zeta_{0}\right)$ of the initial data $\zeta_{0}=\left(\boldsymbol{u}_{0}, \boldsymbol{u}_{1}, \boldsymbol{u}_{2}, \chi_{0}, \chi_{1}\right) \in$ $\mathcal{W} \subset \mathcal{Z}$ is a nonempty, compact and connected subset of $\mathcal{Z}$. Furthermore, $\omega\left(\zeta_{0}\right)$ is positive invariant with respect to $S(t)$, i.e.,

$$
S(t) \omega\left(\zeta_{0}\right) \subset \omega\left(\zeta_{0}\right) \quad \text { for any } t \geq 0 .
$$

(ii) If $\boldsymbol{b}=\mathbf{0}$ then the map $F_{\Omega}: \mathcal{W} \rightarrow \mathbb{R}$ defined by

$F_{\Omega}(\zeta(t))=\int_{\Omega}\left[\frac{1}{2}\left|\boldsymbol{u}_{t}(t)\right|^{2}+W\left(\varepsilon(\boldsymbol{u}(t), \chi(t))+\psi(\chi(t))+\frac{\gamma}{2}|\nabla \chi(t)|^{2}\right] d x\right.$ is the Lyapunov functional for the semigroup $S(t)$, i.e.,

$$
F_{\Omega}\left(S(t) \zeta_{0}\right) \leq F_{\Omega}\left(\zeta_{0}\right) \quad \text { for any } \zeta_{0} \in \mathcal{W}, t \geq 0
$$

$F_{\Omega}$ is constant on the $\omega$-limit set $\omega\left(\zeta_{0}\right)$.

(iii) Every element $\zeta_{\infty}=\left(\boldsymbol{u}_{\infty}, \boldsymbol{u}_{\infty, t}, \boldsymbol{u}_{\infty, t t}, \chi_{\infty}, \chi_{\infty, t}\right)$ of the $\omega$-limit set $\omega\left(\zeta_{0}\right)$ is characterized by

$$
\zeta_{\infty} \equiv\left(\boldsymbol{u}_{\infty}, \mathbf{0}, \mathbf{0}, \chi_{\infty}, 0\right)
$$

with functions $\boldsymbol{u}_{\infty}, \chi_{\infty}$ independent of time, solving the stationary problem corresponding to (1.1)-(1.3):

$$
\begin{array}{rlr}
-\nabla \cdot W_{, \varepsilon}\left(\varepsilon\left(\boldsymbol{u}_{\infty}\right), \chi_{\infty}\right)=\mathbf{0} & \text { a.e. in } \Omega, & \\
\boldsymbol{u}_{\infty}=\mathbf{0} & \text { a.e. on } S, & \\
-\gamma \Delta \chi_{\infty}+\psi^{\prime}\left(\chi_{\infty}\right)+W_{, \chi}\left(\varepsilon\left(\boldsymbol{u}_{\infty}\right), \chi_{\infty}\right)=\bar{\mu} & \text { a.e. in } \Omega, \\
\boldsymbol{n} \cdot \nabla \chi_{\infty} & =0 & \text { a.e. on } S, \\
f_{\Omega} \chi_{\infty} d x & =\chi_{m}:=\int_{\Omega} \chi_{0} d x, &
\end{array}
$$

where $\bar{\mu}$ is a constant to be determined along with the functions $\boldsymbol{u}_{\infty}, \chi_{\infty}$

In the proof of Theorem 2.2 a crucial role is played by uniform in time estimates (2.6) and (2.7). In particular, $L_{2}$ estimates of $\boldsymbol{\varepsilon}\left(\boldsymbol{u}_{t}\right)$ and $\nabla \mu$ on the infinite time interval $(0, \infty)$ (which are due to the mechanical and diffusive dissipation) ensure that $\boldsymbol{u}_{t}$ and $\nabla \mu$ vanish in the limit $t \rightarrow \infty$.

3. Outline of the existence proof. Basic estimates. In this section we present the main ideas of the proof of Theorem 2.1 (see [5] for details) with complementary estimates needed in the study of the asymptotic behaviour.

The proof consists in prolonging the local solution on the intervals $[k T$, $(k+1) T], T>0, k \in \mathbb{N} \cup\{0\}$, up to $k=\infty$. The existence of a local solution 
is obtained by implementing a Galerkin method and passing to the limit with the approximation. A crucial role in prolonging local solutions is played by absorbing type estimates. They have the property of time-decreasing influence of the initial data. We use two kinds of estimates: energy and regularity estimates. The energy estimates are derived on the basis of the original form (1.1)-(1.3) of the system whereas the regularity estimates on the basis of its time-differentiated form.

\subsection{Energy estimates}

3.1.1. Energy identity. A characteristic property of system (1.1)-(1.3) is the mass conservation

$$
\frac{d}{d t} \int_{\Omega} \chi(t) d x=0 \quad \text { for } t>0
$$

which follows from $(1.2)_{1}$ and $(1.2)_{3}$, and shows that the mean value of $\chi$ is preserved, i.e.,

$$
f_{\Omega} \chi(t) d x=f_{\Omega} \chi_{0} d x=: \chi_{m} \quad \text { for } t>0 .
$$

Another property is the energy identity

$$
\begin{aligned}
\frac{d}{d t} F(t)+\nu \int_{\Omega} \boldsymbol{\varepsilon}\left(\boldsymbol{u}_{t}(t)\right) \cdot \boldsymbol{A} \varepsilon\left(\boldsymbol{u}_{t}(t)\right) d x & +\int_{\Omega}|\nabla \mu(t)|^{2} d x \\
& =\int_{\Omega} \boldsymbol{b}(t) \cdot \boldsymbol{u}_{t}(t) d x \quad \text { for } t>0,
\end{aligned}
$$

with the function $F:[0, \infty) \rightarrow[0, \infty)$, given by

$$
F(t)=\int_{\Omega}\left[\frac{1}{2}\left|\boldsymbol{u}_{t}(t)\right|^{2}+W(\varepsilon(\boldsymbol{u}(t)), \chi(t))+\psi(\chi(t))+\frac{\gamma}{2}|\nabla \chi(t)|^{2}\right] d x,
$$

representing the total energy of the system. The two nonnegative integrals on the left-hand side of (3.2) correspond to the mechanical and diffusive dissipation.

Formally, (3.2) results by testing $(1.1)_{1}$ with $\boldsymbol{u}_{t}(t),(1.2)_{1}$ with $\mu(t)$, and $(1.3)_{1}$ with $-\chi_{t}(t)$, integrating over $\Omega$ and by parts, and summing up the resulting identities.

From (3.2) we infer the Lyapunov property: if $\boldsymbol{b}=\mathbf{0}$ then

$$
\frac{d}{d t} F(t) \leq 0
$$

which shows that $F$ is nonincreasing on solution paths, i.e.,

$$
F(t) \leq F(0) \quad \text { for } t \geq 0 .
$$

On account of the structure assumptions (A3)-(A5),

$$
F(t) \geq c_{F}\left(\|\boldsymbol{u}(t)\|_{\boldsymbol{H}_{0}^{1}(\Omega)}^{2}+\left\|\boldsymbol{u}_{t}(t)\right\|_{\boldsymbol{L}_{2}(\Omega)}^{2}+\|\chi(t)\|_{H^{1}(\Omega)}^{2}\right)-c_{F}^{\prime}
$$


with some explicitly computable positive constants $c_{F}$ and $c_{F}^{\prime}$. Hence, $F(t)$ provides estimates for $\left(\boldsymbol{u}(t), \boldsymbol{u}_{t}(t), \chi(t)\right)$ in the energy norms of $\boldsymbol{H}_{0}^{1}(\Omega) \times$ $\boldsymbol{L}_{2}(\Omega) \times H^{1}(\Omega)$. Integrating (3.2) with respect to time from $t=0$ to $t \in$ $(0, \infty)$, we get

$$
\begin{aligned}
\|\boldsymbol{u}\|_{L_{\infty}\left(0, \infty ; \boldsymbol{H}_{0}^{1}(\Omega)\right)}+ & \left\|\boldsymbol{u}_{t}\right\|_{L_{\infty}\left(0, \infty ; \boldsymbol{L}_{2}(\Omega)\right)}+\|\chi\|_{L_{\infty}\left(0, \infty ; H^{1}(\Omega)\right)} \\
& +\left\|\boldsymbol{u}_{t}\right\|_{L_{2}\left(0, \infty ; \boldsymbol{H}_{0}^{1}(\Omega)\right)}+\|\nabla \mu\|_{L_{2}\left(0, \infty ; \boldsymbol{L}_{2}(\Omega)\right)} \leq c_{0}
\end{aligned}
$$

with constant $c_{0}=c\left(\left\|\left(\boldsymbol{u}_{0}, \boldsymbol{u}_{1}, \chi_{0}\right)\right\|_{\boldsymbol{H}_{0}^{1}(\Omega) \times \boldsymbol{L}_{2}(\Omega) \times H^{1}(\Omega)},\|\boldsymbol{b}\|_{L_{1}\left(0, \infty ; \boldsymbol{L}_{2}(\Omega)\right)}\right)$. Since $F(\cdot)$ is continuous on $[0, \infty)$ this shows estimate $(2.6)$.

3.1.2. Additional estimates. From (1.14) it follows, on account of (3.5), that

$$
\left|\int_{\Omega} \mu d x\right| \leq c \int_{\Omega}\left(|\chi|^{3}+|\varepsilon(\boldsymbol{u})|+1\right) d x \leq c\left(c_{0}\right) \quad \text { for } t \geq 0 .
$$

Hence, by the Poincaré inequality, estimates (3.5) and (3.6) imply that for any $t \geq 0$ and any fixed $T>0$,

$$
\begin{aligned}
\|\mu\|_{L_{2}\left(t, t+T ; L_{2}(\Omega)\right)}^{2} & \leq c \int_{t}^{t+T}\left(\|\nabla \mu\|_{\boldsymbol{L}_{2}(\Omega)}^{2}+\left|\int_{\Omega} \mu d x\right|^{2}\right) d t \\
& \leq c\|\nabla \mu\|_{L_{2}\left(t, t+T ; \boldsymbol{L}_{2}(\Omega)\right)}^{2}+c T \sup _{t \in[t, t+T]}\left|\int_{\Omega} \mu d x\right|^{2} \\
& \leq c\left(c_{0}\right)(T+1) .
\end{aligned}
$$

Thus,

$$
\|\mu\|_{L_{2}\left(t, t+T ; H^{1}(\Omega)\right)}^{2} \leq c\left(c_{0}\right)(T+1),
$$

which yields the second estimate in (2.8).

The first estimate in (2.8) follows by testing (1.14) with $\Delta \chi$ and using the Cauchy-Schwarz inequality, which on account of (3.7) and (3.5) yields

$$
\begin{aligned}
\gamma\|\Delta \chi\|_{L_{2}\left(t, t+T ; L_{2}(\Omega)\right)} \leq & \|\mu\|_{L_{2}\left(t, t+T ; L_{2}(\Omega)\right)}+\left\|\chi^{3}-\chi\right\|_{L_{2}\left(t, t+T ; L_{2}(\Omega)\right)} \\
& +c\left(\|\boldsymbol{\varepsilon}(\boldsymbol{u})\|_{L_{2}\left(t, t+T ; \boldsymbol{L}_{2}(\Omega)\right)}+1\right) \leq c\left(c_{0}\right)\left(T^{1 / 2}+1\right) .
\end{aligned}
$$

This together with (3.1), by the ellipticity of the Laplace operator, shows $(2.8)_{1}$.

\subsection{Energy estimates of absorbing type}

3.2.1. A differential inequality for a modified energy function. Let $G$ : $[0, \infty) \rightarrow[0, \infty)$ be defined by

$$
G(t)=F(t)+\frac{\nu c_{*} d_{1}}{2} \int_{\Omega}\left[\boldsymbol{u}_{t}(t) \cdot \boldsymbol{u}(t)+\frac{\nu}{2} \boldsymbol{\varepsilon}(\boldsymbol{u}(t)) \cdot \boldsymbol{A} \boldsymbol{\varepsilon}(\boldsymbol{u}(t))\right] d x
$$


with the constant $c_{*}>0$ given in the coercivity condition (2.1) and $d_{1}>0$ denoting the constant from the Korn inequality

$$
d_{1}^{1 / 2}\|\boldsymbol{u}\|_{\boldsymbol{H}_{0}^{1}(\Omega)} \leq\|\boldsymbol{\varepsilon}(\boldsymbol{u})\|_{\boldsymbol{L}_{2}(\Omega)} \quad \text { for } \boldsymbol{u} \in \boldsymbol{H}_{0}^{1}(\Omega) .
$$

By definition of $G(t)$,

$$
G(t) \geq F(t)-\frac{1}{4}\left\|\boldsymbol{u}_{t}(t)\right\|_{\boldsymbol{L}_{2}(\Omega)}^{2} .
$$

Hence, similarly to $F(t)$, the function $G(t)$ provides estimates on $\left(\boldsymbol{u}, \boldsymbol{u}_{t}, \chi\right)$ in the energy norms of $\boldsymbol{H}_{0}^{1}(\Omega) \times \boldsymbol{L}_{2}(\Omega) \times H^{1}(\Omega)$.

It has been proved (see [5, Lemma 3.3]) that solutions of (1.1)-(1.3) satisfy the differential inequality

$$
\begin{aligned}
& \frac{d}{d t} G(t)+\beta_{1} G(t)+\frac{\nu c_{*} d_{1}}{8}\left\|\boldsymbol{u}_{t}(t)\right\|_{\boldsymbol{H}_{0}^{1}(\Omega)}^{2}+\frac{1}{2}\|\nabla \mu(t)\|_{\boldsymbol{L}_{2}(\Omega)}^{2} \\
& \leq \Lambda_{1}\|\boldsymbol{b}(t)\|_{\boldsymbol{L}_{2}(\Omega)}^{2}+\Lambda_{2} \quad \text { for } t>0,
\end{aligned}
$$

with some explicitly computable positive constants $\beta_{1}, \Lambda_{1}, \Lambda_{2}$.

The proof of (3.9) is based on three identities: the energy identity (3.2), the identity

$$
\gamma \int_{\Omega}|\nabla \chi|^{2} d x+\int_{\Omega}\left[\psi^{\prime}(\chi) \chi+W_{, \chi}(\varepsilon(\boldsymbol{u}), \chi) \chi\right] d x=\int_{\Omega} \mu \chi d x,
$$

resulting from testing equation $(1.3)_{1}$ with $\chi(t)$, and the identity

$$
\begin{aligned}
\frac{d}{d t} \int_{\Omega} \boldsymbol{u}_{t} \cdot \boldsymbol{u} d x+\int_{\Omega} W_{, \varepsilon}(\varepsilon(\boldsymbol{u}), \chi) \cdot \varepsilon(\boldsymbol{u}) d x+\nu & \int_{\Omega} \varepsilon(\boldsymbol{u}) \cdot \boldsymbol{A} \boldsymbol{\varepsilon}\left(\boldsymbol{u}_{t}\right) d x \\
& =\int_{\Omega} \boldsymbol{b} \cdot \boldsymbol{u} d x+\int_{\Omega}\left|\boldsymbol{u}_{t}\right|^{2} d x,
\end{aligned}
$$

following by testing $(1.1)_{1}$ with $\boldsymbol{u}(t)$. An appropriate technical construction based on the structure assumptions on $W(\varepsilon(\boldsymbol{u}), \chi)$ and $\psi(\chi)$ and using straightforward calculations allows us to deduce (3.9) from the abovementioned identities.

3.2.2. Absorbing estimate for $G(t)$. From (3.9) it follows that

$$
G(t) \leq A_{1}\left(1-e^{-\beta_{1} t}\right)+G(0) e^{-\beta_{1} t} \equiv \bar{G}(t), \quad t \geq 0,
$$

where

$$
A_{1}=\frac{1}{\beta_{1}}\left(\Lambda_{1} \sup _{t \in(0, \infty)}\|\boldsymbol{b}(t)\|_{\boldsymbol{L}_{2}(\Omega)}^{2}+\Lambda_{2}\right) .
$$

This estimate is of key importance for prolonging the solution step by step on $[k T,(k+1) T], k \in \mathbb{N} \cup\{0\}$, up to $k=\infty$. In particular, it provides the 
estimate

$$
\begin{aligned}
\|\boldsymbol{u}(t)\|_{\boldsymbol{H}_{0}^{1}(\Omega)}+\left\|\boldsymbol{u}_{t}(t)\right\|_{\boldsymbol{L}_{2}(\Omega)}+\|\chi(t)\|_{H^{1}(\Omega)} & \leq G(t)+c_{F}^{\prime} \\
& \leq A_{1}+G(0)+c_{F}^{\prime} \equiv c_{1}
\end{aligned}
$$

on each time interval $[k T,(k+1) T]$ with constant $c_{1}$ independent of $k$.

3.2.3. Absorbing set in energy norms. Inequality (3.10) implies that

$$
\limsup _{t \rightarrow \infty} G(t) \leq A_{1}
$$

Let us consider two cases:

$$
A_{1}<G(0) \text { and } A_{1} \geq G(0) .
$$

In the first case

$$
\bar{G}^{\prime}(t)=\left(A_{1}-G(0)\right) \beta_{1} e^{-\beta_{1} t}<0,
$$

so $\bar{G}(t)$ decreases from $G(0)$ to $A_{1}$. Let $A_{1}^{\prime}$ be any positive number such that $A_{1}^{\prime}>A_{1}$. Then there exists a time $t_{1}=t_{1}\left(G(0), A_{1}^{\prime}\right)$ given by

$$
t_{1}=\frac{1}{\beta_{1}} \log \frac{G(0)}{A_{1}^{\prime}-A_{1}}
$$

such that $G(t)<A_{1}^{\prime}$ for all $t \geq t_{1}$. Hence

$$
\begin{aligned}
\|\boldsymbol{u}(t)\|_{\boldsymbol{H}_{0}^{1}(\Omega)}+\left\|\boldsymbol{u}_{t}(t)\right\|_{\boldsymbol{L}_{2}(\Omega)}+ & \|\chi(t)\|_{H^{1}(\Omega)} \\
& <A_{1}^{\prime}+c_{F}^{\prime} \equiv c_{1 a} \quad \text { for all } t \geq t_{1} .
\end{aligned}
$$

This shows the absorbing set for $\left(\boldsymbol{u}, \boldsymbol{u}_{t}, \chi\right)$ in the energy norms of $\boldsymbol{H}_{0}^{1}(\Omega) \times$ $\boldsymbol{L}_{2}(\Omega) \times H^{1}(\Omega)$.

In the second case $\left(A_{1} \geq G(0)\right), \bar{G}^{\prime}(t)>0$ so $\bar{G}(t)$ is increasing from $G(0)$ to $A_{1}$. Hence (3.12) is automatically satisfied.

\subsection{Regularity estimates of absorbing type}

3.3.1. A differential inequality in higher norms. Let $N:[0, \infty) \rightarrow[0, \infty)$ be the function constructed from a regular solution, defined as a linear combination with appropriately chosen coefficients (depending on the constant $c_{1}$ in $\left.(3.11)\right)$ of the modified energy $G(t)$ and the norms

$$
\begin{array}{llll}
\|\boldsymbol{Q u}(t)\|_{\boldsymbol{L}_{2}(\Omega)}^{2}, & \left\|\boldsymbol{Q}^{1 / 2} \boldsymbol{u}_{t}(t)\right\|_{\boldsymbol{L}_{2}(\Omega)}^{2}, & \left\|\boldsymbol{Q} \boldsymbol{u}_{t}(t)\right\|_{\boldsymbol{L}_{2}(\Omega)}^{2}, & \left\|\boldsymbol{Q}^{1 / 2} \boldsymbol{u}_{t t}(t)\right\|_{\boldsymbol{L}_{2}(\Omega)}^{2}, \\
\|\chi(t)\|_{L_{2}(\Omega)}^{2}, & \|\Delta \chi(t)\|_{L_{2}(\Omega)}^{2}, & \left\|\chi \chi_{t}(t)\right\|_{L_{2}(\Omega)}^{2} .
\end{array}
$$

Here $\boldsymbol{Q}^{1 / 2}$ stands for the fractional power of the operator $\boldsymbol{Q}$ with the domain $D\left(\boldsymbol{Q}^{1 / 2}\right)=\boldsymbol{H}_{0}^{1}(\Omega)$, satisfying

$$
\begin{aligned}
\left\|\boldsymbol{Q}^{1 / 2} \boldsymbol{u}\right\|_{\boldsymbol{L}_{2}(\Omega)}^{2} & =(-\boldsymbol{Q u}, \boldsymbol{u})_{\boldsymbol{L}_{2}(\Omega)} \\
& =\bar{\mu}\|\nabla \boldsymbol{u}\|_{\boldsymbol{L}_{2}(\Omega)}^{2}+(\bar{\lambda}+\bar{\mu})\|\nabla \cdot \boldsymbol{u}\|_{L_{2}(\Omega)}^{2} \quad \text { for } \boldsymbol{u} \in D(\boldsymbol{Q}) .
\end{aligned}
$$


By construction, the function $N(t)$ satisfies the bound

$$
\begin{aligned}
N(t) \geq & c_{N}\left(\|\boldsymbol{u}(t)\|_{\boldsymbol{H}^{2}(\Omega)}^{2}+\left\|\boldsymbol{u}_{t}(t)\right\|_{\boldsymbol{H}^{2}(\Omega)}^{2}+\left\|\boldsymbol{u}_{t t}(t)\right\|_{\boldsymbol{H}_{0}^{1}(\Omega)}^{2}\right. \\
& \left.+\|\chi(t)\|_{H_{N}^{2}(\Omega)}^{2}+\left\|\chi_{t}(t)\right\|_{L_{2}(\Omega)}^{2}\right)-c_{N}^{\prime}
\end{aligned}
$$

with explicitly computable, positive constants $c_{N}$ and $c_{N}^{\prime}$ depending on $c_{1}$. Thus, $N(t)$ provides estimates for $\left(\boldsymbol{u}(t), \boldsymbol{u}_{t}(t), \boldsymbol{u}_{t t}(t), \chi(t), \chi_{t}(t)\right)$ in the norms of $\boldsymbol{H}^{2}(\Omega) \times \boldsymbol{H}^{2}(\Omega) \times \boldsymbol{H}_{0}^{1}(\Omega) \times H_{N}^{2}(\Omega) \times L_{2}(\Omega)$.

It has been proved in [5, Lemma 4.5] that solutions of (1.1)-(1.3) satisfy the differential inequality

$$
\begin{aligned}
\frac{d}{d t} N(t)+\beta_{5} N(t)+\tilde{\beta}_{5} \tilde{N}(t) & \\
& \leq \Lambda_{3}\|\boldsymbol{b}(t)\|_{\boldsymbol{L}_{2}(\Omega)}^{2}+\Lambda_{4}\left\|\boldsymbol{b}_{t}(t)\right\|_{\boldsymbol{L}_{2}(\Omega)}^{2}+\Lambda_{5} \quad \text { for } t>0
\end{aligned}
$$

where

$$
\tilde{N}(t)=\left\|\boldsymbol{u}_{t t}(t)\right\|_{\boldsymbol{H}^{2}(\Omega)}^{2}+\left\|\chi_{t}(t)\right\|_{H^{2}(\Omega)}^{2},
$$

and $\beta_{5}, \tilde{\beta}_{5}, \Lambda_{3}, \Lambda_{4}, \Lambda_{5}$ are explicitly computable positive constants depending on $c_{1}$.

Derivation of that inequality is based on differentiating system (1.12)(1.14) with respect to time. A straightforward but technical procedure consists of the following main steps. In the first step we derive a differential inequality corresponding to the elasticity system (1.12):

$$
\begin{aligned}
\frac{d}{d t} H(t)+ & \beta_{2} H(t)+\tilde{\beta}_{2} \tilde{H}(t) \\
\leq & c_{H}\left(\|\nabla \chi(t)\|_{\boldsymbol{L}_{2}(\Omega)}^{2}+\left\|\chi_{t}(t) \nabla \chi(t)\right\|_{\boldsymbol{L}_{2}(\Omega)}^{2}+\left\|\nabla \chi_{t}(t)\right\|_{\boldsymbol{L}_{2}(\Omega)}^{2}\right. \\
& \left.+\|\boldsymbol{b}(t)\|_{\boldsymbol{L}_{2}(\Omega)}^{2}+\left\|\boldsymbol{b}_{t}(t)\right\|_{\boldsymbol{L}_{2}(\Omega)}^{2}\right) \quad \text { for } t>0
\end{aligned}
$$

where $H:[0, \infty) \rightarrow[0, \infty)$ is a linear combination of the norms

$$
\|\boldsymbol{Q u}(t)\|_{\boldsymbol{L}_{2}(\Omega)}^{2}, \quad\left\|\boldsymbol{Q}^{1 / 2} \boldsymbol{u}_{t}(t)\right\|_{\boldsymbol{L}_{2}(\Omega)}^{2}, \quad\left\|\boldsymbol{Q} \boldsymbol{u}_{t}(t)\right\|_{\boldsymbol{L}_{2}(\Omega)}^{2}, \quad\left\|\boldsymbol{Q}^{1 / 2} \boldsymbol{u}_{t t}(t)\right\|_{\boldsymbol{L}_{2}(\Omega)}^{2},
$$

and

$$
\tilde{H}(t)=\left\|\boldsymbol{u}_{t t}(t)\right\|_{\boldsymbol{L}_{2}(\Omega)}^{2}+\left\|\boldsymbol{Q} \boldsymbol{u}_{t t}(t)\right\|_{\boldsymbol{L}_{2}(\Omega)}^{2},
$$

and $\beta_{2}, \tilde{\beta}_{2}, c_{H}$ are explicitly computable positive constants.

In the second step we derive a differential inequality corresponding to system (1.13), (1.14) which allows one to handle the terms on the right-hand side of (3.15). The inequality has the form

$$
\frac{d}{d t} J(t)+\beta_{3} J(t)+\tilde{\beta}_{3} \tilde{J}(t) \leq c_{J}\left(\left\|\boldsymbol{\varepsilon}\left(\boldsymbol{u}_{t}(t)\right)\right\|_{\boldsymbol{L}_{2}(\Omega)}^{2}+\chi_{m}^{2}+1\right) \quad \text { for } t>0,
$$

where $J:[0, \infty) \rightarrow[0, \infty)$ is a linear combination of the norms

$$
\|\chi(t)\|_{L_{2}(\Omega)}^{2}, \quad\|\Delta \chi(t)\|_{L_{2}(\Omega)}^{2}, \quad\left\|\chi_{t}(t)\right\|_{L_{2}(\Omega)}^{2},
$$


and

$$
\tilde{J}(t)=\left\|\chi_{t}(t)\right\|_{H^{2}(\Omega)}^{2},
$$

and $\beta_{3}, \tilde{\beta}_{3}, c_{J}$ are positive constants.

In the third step we combine (3.15) and (3.16) to deduce the differential inequality

$$
\begin{aligned}
& \frac{d}{d t} K(t)+\beta_{4} K(t)+\tilde{\beta}_{4} \tilde{K}(t) \\
\leq & c_{K}\left(\left\|\boldsymbol{\varepsilon}\left(\boldsymbol{u}_{t}(t)\right)\right\|_{\boldsymbol{L}_{2}(\Omega)}^{2}+\|\boldsymbol{b}(t)\|_{\boldsymbol{L}_{2}(\Omega)}^{2}+\left\|\boldsymbol{b}_{t}(t)\right\|_{\boldsymbol{L}_{2}(\Omega)}^{2}+1\right) \quad \text { for } t>0,
\end{aligned}
$$

where $K:[0, \infty) \rightarrow[0, \infty)$ is a linear combination of the terms of $H(t)$ and $J(t)$; moreover,

$$
\tilde{K}(t)=\tilde{H}(t)+\tilde{J}(t),
$$

and $\beta_{4}, \tilde{\beta}_{4}, c_{K}$ are positive constants.

Finally, combining inequalities (3.17) and (3.9) allows us to absorb the term $\| \varepsilon\left(\boldsymbol{u}_{t}(t) \|_{\boldsymbol{L}_{2}(\Omega)}^{2}\right.$ on the right-hand side of (3.17) and thereby deduce (3.14).

3.3.2. Absorbing estimate for $N(t)$. On account of (3.14) we have

$$
N(t) \leq A_{2}\left(1-e^{-\beta_{5} t}\right)+N(0) e^{-\beta_{5} t}
$$

where

$$
A_{2}=\frac{1}{\beta_{5}}\left(\Lambda_{3} \sup _{t \in(0, \infty)}\|\boldsymbol{b}(t)\|_{L_{2}(\Omega)}^{2}+\Lambda_{4} \sup _{t \in(0, \infty)}\left\|\boldsymbol{b}_{t}(t)\right\|_{L_{2}(\Omega)}^{2}+\Lambda_{5}\right) .
$$

Estimate (3.18) allows one to prolong a regular solution step by step on the intervals $[k T,(k+1) T], k \in \mathbb{N}$. It provides the following bound uniform in $k$ :

$$
\sup _{k \in \mathbb{N} \cup\{0\}} \max _{t \in[k T,(k+1) T]} N(t) \leq A_{2}+N(0) .
$$

Moreover, by integrating (3.14) with respect to time, it follows that

$$
\sup _{k \in \mathbb{N} \cup\{0\}} \int_{k T}^{(k+1) T} \tilde{\beta}_{5} \tilde{N}(t) d t \leq T A_{2} \beta_{5}+A_{2}+N(0) .
$$

In view of the definitions of $N(t)$ and $\tilde{N}(t)$, estimates (3.19) and (3.20) imply the corresponding bounds on $\boldsymbol{u}$ and $\chi$ in (2.7)-(2.9).

Furthermore, testing $(1.2)_{1}$ with $\Delta \mu$ and using estimate (2.7) on $\chi_{t}$ gives

$$
\|\Delta \mu\|_{C\left([0, \infty) ; L_{2}(\Omega)\right)} \leq c .
$$

Hence, recalling (3.6), the ellipticity of the Laplace operator with homogeneous boundary condition implies that

$$
\|\mu\|_{C\left([0, \infty) ; H_{N}^{2}(\Omega)\right)} \leq c\left(\|\Delta \mu\|_{C\left([0, \infty) ; L_{2}(\Omega)\right)}+\sup _{t \in[0, \infty)}\left|\int_{\Omega} \mu d x\right|\right) \leq c,
$$

which provides the estimate on $\mu$ in (2.7). 
3.3.3. Additional estimates. On the basis of (2.6)-(2.9) we can deduce additional estimates (2.10) which are used in the long-time analysis. The estimate on $\mu_{t}$ in (2.10) follows from the identity (resulting by differentiating $(1.14)_{1}$ with respect to time)

$$
\begin{aligned}
\mu_{t}= & -\gamma \Delta \chi_{t}+\psi^{\prime \prime}(\chi) \chi_{t}+z^{\prime \prime}(\chi) \chi_{t}(\boldsymbol{B} \cdot \boldsymbol{\varepsilon}(\boldsymbol{u})+D z(\chi)+E) \\
& +z^{\prime}(\chi)\left(\boldsymbol{B} \cdot \boldsymbol{\varepsilon}\left(\boldsymbol{u}_{t}\right)+D z^{\prime}(\chi) \chi_{t}\right)
\end{aligned}
$$

by testing it with $\mu_{t}$ and applying the Cauchy-Schwarz inequality. Then

$$
\begin{aligned}
\left\|\mu_{t}\right\|_{L_{2}\left(t, t+T ; L_{2}(\Omega)\right)} \leq & c\left(\left\|\Delta \chi_{t}\right\|_{L_{2}\left(t, t+T ; L_{2}(\Omega)\right)}+\left\|\chi^{2} \chi_{t}\right\|_{L_{2}\left(t, t+T ; L_{2}(\Omega)\right.}\right. \\
& +\left\|\chi_{t}\right\|_{L_{2}\left(t, t+T ; L_{2}(\Omega)\right)}+\left\|\chi_{t} \boldsymbol{\varepsilon}(\boldsymbol{u})\right\|_{L_{2}\left(t, t+T ; \boldsymbol{L}_{2}(\Omega)\right)} \\
& \left.+\left\|\boldsymbol{\varepsilon}\left(\boldsymbol{u}_{t}\right)\right\|_{L_{2}\left(t, t+T ; \boldsymbol{L}_{2}(\Omega)\right)}\right) \leq c\left(T^{1 / 2}+1\right)
\end{aligned}
$$

where we used (2.7) and (2.9), in particular the bounds

$$
\|\chi\|_{L_{2}\left(t, t+T ; L_{\infty}(\Omega)\right)} \leq c T^{1 / 2}, \quad\left\|\chi_{t}\right\|_{L_{2}\left(t, t+T ; L_{\infty}(\Omega)\right)} \leq c\left(T^{1 / 2}+1\right) .
$$

Estimate on $\boldsymbol{u}_{t t t}$ in (2.10) follows from equation $(1.12)_{1}$ differentiated with respect to $t$. Then for any test function $\boldsymbol{\eta} \in L_{2}\left(t, t+T ; \boldsymbol{H}^{1}(\Omega)\right)$,

$$
\begin{aligned}
&\left|\int_{t}^{t+T}\left(\boldsymbol{u}_{t t t}, \boldsymbol{\eta}\right)_{\boldsymbol{L}_{2}(\Omega)} d t^{\prime}\right|=\mid \int_{t}^{t+T}\left[-\left(\boldsymbol{A} \boldsymbol{\varepsilon}\left(\boldsymbol{u}_{t}\right)+\nu \boldsymbol{A} \boldsymbol{\varepsilon}\left(\boldsymbol{u}_{t t}\right), \boldsymbol{\varepsilon}(\boldsymbol{\eta})\right)_{\boldsymbol{L}_{2}(\Omega)}\right. \\
&\left.\quad+\left(z^{\prime \prime}(\chi) \chi_{t} \boldsymbol{B} \nabla \chi+z^{\prime}(\chi) \boldsymbol{B} \nabla \chi_{t}, \boldsymbol{\eta}\right)_{\boldsymbol{L}_{2}(\Omega)}+\left(\boldsymbol{b}_{t}, \boldsymbol{\eta}\right)_{\boldsymbol{L}_{2}(\Omega)}\right] d t^{\prime} \mid \\
& \leq c\left(\left\|\boldsymbol{\varepsilon}\left(\boldsymbol{u}_{t}\right)\right\|_{L_{2}\left(t, t+T ; \boldsymbol{L}_{2}(\Omega)\right)}+\left\|\boldsymbol{\varepsilon}\left(\boldsymbol{u}_{t t}\right)\right\|_{L_{2}\left(t, t+T ; \boldsymbol{L}_{2}(\Omega)\right)}\right)\|\nabla \boldsymbol{\eta}\|_{L_{2}\left(t, t+T ; \boldsymbol{L}_{2}(\Omega)\right)} \\
&+c\left(\left\|\chi_{t} \nabla \chi\right\|_{L_{2}\left(t, t+T ; \boldsymbol{L}_{2}(\Omega)\right)}+\left\|\nabla \chi_{t}\right\|_{L_{2}\left(t, t+T ; \boldsymbol{L}_{2}(\Omega)\right)}\right. \\
&\left.+\left\|\boldsymbol{b}_{t}\right\|_{L_{2}\left(t, t+T ; \boldsymbol{L}_{2}(\Omega)\right)}\right)\|\boldsymbol{\eta}\|_{L_{2}\left(t, t+T ; \boldsymbol{L}_{2}(\Omega)\right)} \\
& \leq c\left(T^{1 / 2}+1\right)\|\boldsymbol{\eta}\|_{L_{2}\left(t, t+T ; \boldsymbol{H}^{1}(\Omega)\right)}
\end{aligned}
$$

where we used (2.7) and (2.9). This proves $(2.10)_{1}$.

Similarly, by testing equation $(1.13)_{1}$ differentiated with respect to time with a function $\xi \in L_{2}\left(0, T ; H_{N}^{2}(\Omega)\right)$, and using (3.21), we get

$$
\begin{aligned}
& \left|\int_{t}^{t+T}\left(\chi_{t t}, \xi\right)_{L_{2}(\Omega)} d t^{\prime}\right|=\left|\int_{t}^{t+T}\left(\mu_{t}, \Delta \xi\right)_{L_{2}(\Omega)} d t^{\prime}\right| \\
& \quad \leq\left\|\mu_{t}\right\|_{L_{2}\left(t, t+T ; L_{2}(\Omega)\right)}\|\xi\|_{L_{2}\left(t, t+T ; H_{N}^{2}(\Omega)\right)} \leq c\left(T^{1 / 2}+1\right)\|\xi\|_{L_{2}\left(t, t+T ; H_{N}^{2}(\Omega)\right)} .
\end{aligned}
$$

This proves $(2.10)_{2}$.

3.3.4. Absorbing set in stronger norms. For completeness we also recall (see [5, Th. 2.2]) the absorbing set in the norms induced by the function $N(t)$. 
On account of the absorbing estimate (3.12) in energy norms one can infer from inequality (3.18) that for all $t \geq t_{1}$,

$$
N(t) \leq A_{2 a}\left(1-e^{-\beta_{5 a} t}\right)+N(0) e^{-\beta_{5 a} t}
$$

where $\beta_{5 a}$ and $A_{2 a}$ are positive constants independent of the initial condition $N(0)$, obtained by replacing, in appropriate expressions, the constant $c_{1}$ from (3.11) by $c_{1 a}$ from (3.12). From (3.22) it follows that

$$
\limsup _{t \rightarrow \infty} N(t) \leq A_{2 a} .
$$

Thus, for any positive number $A_{2}^{\prime}$ satisfying $A_{2}^{\prime}>A_{2 a}$, there exists a time $t_{2}=t_{2}\left(N(0), A_{2}^{\prime}\right)$,

$$
t_{2}=\frac{1}{\beta_{5 a}} \log \frac{N(0)}{A_{2}^{\prime}-A_{2 a}},
$$

such that $N(t)<A_{2}^{\prime}$ for all $t \geq t_{*}=\max \left\{t_{1}, t_{2}\right\}$. Hence, by (3.13),

$$
\begin{aligned}
c_{N a}\left(\|\boldsymbol{u}(t)\|_{\boldsymbol{H}^{2}(\Omega)}^{2}+\left\|\boldsymbol{u}_{t}(t)\right\|_{\boldsymbol{H}^{2}(\Omega)}^{2}+\left\|\boldsymbol{u}_{t t}(t)\right\|_{\boldsymbol{H}_{0}^{1}(\Omega)}^{2}\right. & \\
& \left.+\|\chi(t)\|_{H_{N}^{2}(\Omega)}^{2}+\left\|\chi_{t}(t)\right\|_{L_{2}(\Omega)}^{2}\right)<A_{2}^{\prime}+c_{N a}^{\prime} \quad \text { for all } t \geq t_{*}
\end{aligned}
$$

where $c_{N a}$ and $c_{N a}^{\prime}$ are positive numbers independent of $N(0)$. This establishes the absorbing set for $\left(\boldsymbol{u}, \boldsymbol{u}_{t}, \boldsymbol{u}_{t t}, \chi, \chi_{t}\right)$ in $\boldsymbol{H}^{2}(\Omega) \times \boldsymbol{H}^{2}(\Omega) \times \boldsymbol{H}_{0}^{1}(\Omega) \times$ $H_{N}^{2}(\Omega) \times L_{2}(\Omega)$.

4. Proof of Theorem 2.2. (i) Due to (2.7) the orbit $\bigcup_{t \geq 0} S(t) \zeta_{0}$ starting at $\zeta_{0}=\left(\boldsymbol{u}_{0}, \boldsymbol{u}_{1}, \boldsymbol{u}_{2}, \chi_{0}, \chi_{1}\right)$ is bounded in the space $\mathcal{W}$, thus it is relatively compact in $\mathcal{Z}$. Hence, the $\omega$-limit set $\omega\left(\zeta_{0}\right)$ is a nonempty and compact subset of $\mathcal{Z}$. Moreover, since by (2.3),

$$
\left(\boldsymbol{u}, \boldsymbol{u}_{t}, \boldsymbol{u}_{t t}, \chi, \chi_{t}\right) \in C([0, \infty) ; \mathcal{W}) \subset C([0, \infty) ; \mathcal{Z}),
$$

the known results of the theory of dynamical systems (see e.g. [3, Prop. 2.1]) show that this set is connected in $\mathcal{Z}$, and positive invariant with respect to $S(t)$. Indeed, if $\zeta \in \omega\left(\zeta_{0}\right)$, say $\zeta=\lim _{n \rightarrow \infty} S\left(t_{n}\right) \zeta_{0}$, then

$$
S(t) \zeta=\lim _{n \rightarrow \infty} S(t) S\left(t_{n}\right) \zeta_{0}=\lim _{n \rightarrow \infty} S\left(t+t_{n}\right) \zeta_{0} \in \omega\left(\zeta_{0}\right) .
$$

(ii) The map $F_{\Omega}$ given by (2.14) coincides with the function $F(t)$ defined in the energy identity (3.2). Thus, the Lyapunov property of $F_{\Omega}$ results immediately from (3.3).

The claim that $F_{\Omega}$ is constant on $\omega\left(\zeta_{0}\right)$ follows from a general result in [3, Proposition 2.2], due to the continuity of $F_{\Omega}$. Indeed, let $F_{\Omega \infty}:=$ $\lim _{t \rightarrow \infty} F_{\Omega}\left(S(t) \zeta_{0}\right)$. Choosing any $\zeta \in \omega\left(\zeta_{0}\right)$, say $\zeta=\lim _{n \rightarrow \infty} S\left(t_{n}\right) \zeta_{0}$, we deduce by the continuity of $F_{\Omega}$ that

$$
F_{\Omega}(\zeta)=F_{\Omega}\left(\lim _{n \rightarrow \infty} S\left(t_{n}\right) \zeta_{0}\right)=\lim _{n \rightarrow \infty} F_{\Omega}\left(S\left(t_{n}\right) \zeta_{0}\right)=F_{\Omega \infty},
$$

which implies that $F_{\Omega}$ is constant on $\omega\left(\zeta_{0}\right)$. 
(iii) We shall characterize the elements of the $\omega$-limit set. Let $\left(\boldsymbol{u}_{\infty}, \boldsymbol{u}_{\infty, t}\right.$, $\left.\boldsymbol{u}_{\infty, t t}, \chi_{\infty}, \chi_{\infty, t}\right) \in \omega\left(\boldsymbol{u}_{0}, \boldsymbol{u}_{1}, \boldsymbol{u}_{2}, \chi_{0}, \chi_{1}\right)$, and $t_{n}$ be a sequence of positive numbers such that $t_{n} \rightarrow \infty$ and

$$
\begin{aligned}
\left(\boldsymbol{u}\left(t_{n}\right), \boldsymbol{u}_{t}\left(t_{n}\right), \boldsymbol{u}_{t t}\left(t_{n}\right), \chi\left(t_{n}\right), \chi_{t}\left(t_{n}\right)\right) & \\
& \rightarrow\left(\boldsymbol{u}_{\infty}, \boldsymbol{u}_{\infty, t}, \boldsymbol{u}_{\infty, t t}, \chi_{\infty}, \chi_{\infty, t}\right) \quad \text { strongly in } \mathcal{Z} .
\end{aligned}
$$

For a fixed number $T>0$ and $t \in[0, T]$ we define the functions

$$
\begin{aligned}
\boldsymbol{u}_{n}(t):=\boldsymbol{u}\left(t_{n}+t\right), & \boldsymbol{u}_{n, t}(t):=\boldsymbol{u}_{t}\left(t_{n}+t\right), & \boldsymbol{u}_{n, t t}(t):=\boldsymbol{u}_{t t}\left(t_{n}+t\right), \\
\chi_{n}(t):=\chi\left(t_{n}+t\right), & \chi_{n, t}(t):=\chi_{t}\left(t_{n}+t\right), & \mu_{n}(t):=\mu\left(t_{n}+t\right), \\
\boldsymbol{b}_{n}(t):=\boldsymbol{b}\left(t_{n}+t\right), & &
\end{aligned}
$$

where $(\boldsymbol{u}, \chi, \mu)$ is the solution of $(1.1)-(1.3)$ on $[0, \infty)$. Thus, $\left(\boldsymbol{u}_{n}, \chi_{n}, \mu_{n}\right)$ solve the system

$$
\begin{aligned}
& \boldsymbol{u}_{n, t t}-\boldsymbol{Q} \boldsymbol{u}_{n}-\nu \boldsymbol{Q} \boldsymbol{u}_{n, t} \\
& =z^{\prime}\left(\chi_{n}\right) \boldsymbol{B} \nabla \chi_{n}+\boldsymbol{b}_{n} \quad \text { in } \Omega^{T}=\Omega \times(0, T), \\
& \boldsymbol{u}_{n}(0)=\boldsymbol{u}\left(t_{n}\right), \quad \boldsymbol{u}_{n, t}(0)=\boldsymbol{u}_{t}\left(t_{n}\right) \quad \text { in } \Omega \text {, } \\
& \boldsymbol{u}_{n}=\mathbf{0} \\
& \text { on } S^{T}=S \times(0, T) \text {, } \\
& \chi_{n, t}-\Delta \mu_{n}=0 \quad \text { in } \Omega^{T}, \\
& \chi_{n}(0)=\chi\left(t_{n}\right) \quad \text { in } \Omega \text {, } \\
& \boldsymbol{n} \cdot \nabla \mu_{n}=0 \quad \text { on } S^{T} \text {, } \\
& \mu_{n}=-\gamma \Delta \chi_{n}+\psi^{\prime}\left(\chi_{n}\right) \\
& +z^{\prime}\left(\chi_{n}\right)\left(\boldsymbol{B} \cdot \boldsymbol{\varepsilon}\left(\boldsymbol{u}_{n}\right)+D z\left(\chi_{n}\right)+E\right) \text { in } \Omega^{T}, \\
& \boldsymbol{n} \cdot \nabla \chi_{n}=0 \quad \text { on } S^{T} \text {. }
\end{aligned}
$$

By virtue of (2.6) and (2.7) the following estimates hold true independently of $T$ and $n$ :

$$
\begin{aligned}
\left\|\boldsymbol{u}_{n}\right\|_{L_{\infty}\left(0, T ; \boldsymbol{H}_{0}^{1}(\Omega)\right)}+ & \left\|\boldsymbol{u}_{n, t}\right\|_{L_{\infty}\left(0, T ; \boldsymbol{L}_{2}(\Omega)\right)}+\left\|\chi_{n}\right\|_{L_{\infty}\left(0, T ; H^{1}(\Omega)\right)} \\
& +\left\|\boldsymbol{u}_{n, t}\right\|_{L_{2}\left(0, T ; \boldsymbol{H}_{0}^{1}(\Omega)\right)}+\|\nabla \mu\|_{L_{2}\left(0, T ; \boldsymbol{L}_{2}(\Omega)\right)} \leq c_{0}, \\
\left\|\boldsymbol{u}_{n}\right\|_{W_{\infty}^{1}\left(0, T ; \boldsymbol{H}^{2}(\Omega)\right)}+ & \left\|\boldsymbol{u}_{n, t t}\right\|_{L_{\infty}\left(0, T ; \boldsymbol{H}_{0}^{1}(\Omega)\right)}+\left\|\chi_{n}\right\|_{L_{\infty}\left(0, T ; H_{N}^{2}(\Omega)\right)} \\
& +\left\|\chi_{n, t}\right\|_{L_{\infty}\left(0, T ; L_{2}(\Omega)\right)}+\left\|\mu_{n}\right\|_{L_{\infty}\left(0, T ; H_{N}^{2}(\Omega)\right)} \leq c .
\end{aligned}
$$

Moreover, by (2.8)-(2.10),

$$
\begin{gathered}
\left\|\chi_{n}\right\|_{L_{2}\left(0, T ; H_{N}^{2}(\Omega)\right)}+\left\|\mu_{n}\right\|_{L_{2}\left(0, T ; H^{1}(\Omega)\right)} \leq c(T), \\
\left\|\boldsymbol{u}_{n, t t}\right\|_{L_{2}\left(0, T ; H^{2}(\Omega)\right)}+\left\|\chi_{n, t}\right\|_{L_{2}\left(0, T ; H_{N}^{2}(\Omega)\right)} \leq c(T),
\end{gathered}
$$




$$
\begin{aligned}
\left\|\boldsymbol{u}_{n, t t t}\right\|_{L_{2}\left(0, T ;\left(\boldsymbol{H}_{0}^{1}(\Omega)\right)^{\prime}\right)}+\left\|\chi_{n, t t}\right\|_{L_{2}\left(0, T ;\left(H_{N}^{2}(\Omega)\right)^{\prime}\right)} \\
+\left\|\mu_{n, t}\right\|_{L_{2}\left(0, T ; L_{2}(\Omega)\right)} \leq c(T)
\end{aligned}
$$

with a constant $c(T)$ depending on $T$ but not on $n$.

The above estimates allow us to pass to the weak limit $n \rightarrow \infty$ in $(4.3)-$ (4.5). In fact, it follows from (4.6)-(4.10) that there exist functions $(\overline{\boldsymbol{u}}, \bar{\chi}, \bar{\mu})$ with

$$
\begin{aligned}
& \overline{\boldsymbol{u}} \in W_{\infty}^{1}\left(0, T ; \boldsymbol{H}^{2}(\Omega) \cap \boldsymbol{H}_{0}^{1}(\Omega)\right), \\
& \overline{\boldsymbol{u}}_{t t} \in L_{\infty}\left(0, T ; \boldsymbol{H}_{0}^{1}(\Omega)\right) \cap L_{2}\left(0, T ; \boldsymbol{H}^{2}(\Omega)\right), \\
& \overline{\boldsymbol{u}}_{t t t} \in L_{2}\left(0, T ;\left(\boldsymbol{H}_{0}^{1}(\Omega)\right)^{\prime}\right), \quad \bar{\chi} \in L_{\infty}\left(0, T ; H_{N}^{2}(\Omega)\right), \\
& \bar{\chi}_{t} \in L_{\infty}\left(0, T ; L_{2}(\Omega)\right) \cap L_{2}\left(0, T ; H_{N}^{2}(\Omega)\right), \\
& \bar{\chi}_{t t} \in L_{2}\left(0, T ;\left(H_{N}^{2}(\Omega)\right)^{\prime}\right), \\
& \bar{\mu} \in L_{\infty}\left(0, T ; H_{N}^{2}(\Omega)\right), \quad \bar{\mu}_{t} \in L_{2}\left(0, T ; L_{2}(\Omega)\right),
\end{aligned}
$$

and subsequences of $\left(\boldsymbol{u}_{n}, \chi_{n}, \mu_{n}\right)$ (which we still denote by the same indices) such that as $n \rightarrow \infty$,

$$
\begin{aligned}
& \boldsymbol{u}_{n} \rightarrow \overline{\boldsymbol{u}} \quad \text { weakly* }^{*} \text { in } W_{\infty}^{1}\left(0, T ; \boldsymbol{H}^{2}(\Omega)\right), \\
& \boldsymbol{u}_{n, t t} \rightarrow \overline{\boldsymbol{u}}_{t t} \quad \text { weakly* }^{*} \text { in } L_{\infty}\left(0, T ; \boldsymbol{H}_{0}^{1}(\Omega)\right) \\
& \text { and weakly in } L_{2}\left(0, T ; \boldsymbol{H}^{2}(\Omega)\right) \text {, } \\
& \boldsymbol{u}_{n, t t t} \rightarrow \overline{\boldsymbol{u}}_{t t t} \quad \text { weakly in } L_{2}\left(0, T ;\left(\boldsymbol{H}_{0}^{1}(\Omega)\right)^{\prime}\right) \text {, } \\
& \chi_{n} \rightarrow \bar{\chi} \quad \text { weakly* in } L_{\infty}\left(0, T ; H_{N}^{2}(\Omega)\right) \text {, } \\
& \chi_{n, t} \rightarrow \bar{\chi}_{t} \quad \text { weakly* }^{*} \text { in } L_{\infty}\left(0, T ; L_{2}(\Omega)\right) \\
& \text { and weakly in } L_{2}\left(0, T ; H_{N}^{2}(\Omega)\right) \text {, } \\
& \chi_{n, t t} \rightarrow \bar{\chi}_{t t} \quad \text { weakly in } L_{2}\left(0, T ;\left(H_{N}^{2}(\Omega)\right)^{\prime}\right) \text {, } \\
& \mu_{n} \rightarrow \bar{\mu} \quad \text { weakly* in } L_{\infty}\left(0, T ; H_{N}^{2}(\Omega)\right) \text {, } \\
& \mu_{n, t} \rightarrow \bar{\mu}_{t} \quad \text { weakly in } L_{2}\left(0, T ; L_{2}(\Omega)\right) .
\end{aligned}
$$

Hence, by the standard compactness results (see e.g. [6]) it follows in particular that for $n \rightarrow \infty$,

$$
\begin{aligned}
& \boldsymbol{u}_{n} \rightarrow \overline{\boldsymbol{u}}, \quad \boldsymbol{u}_{n, t} \rightarrow \overline{\boldsymbol{u}}_{t} \quad \text { strongly in } C\left([0, T] ; \boldsymbol{H}_{0}^{1}(\Omega)\right) \\
& \text { and a.e. in } \Omega^{T} \text {, } \\
& \boldsymbol{u}_{n, t t} \rightarrow \overline{\boldsymbol{u}}_{t t} \quad \text { strongly in } L_{2}\left(0, T ; \boldsymbol{H}_{0}^{1}(\Omega)\right) \cap C\left([0, T] ; \boldsymbol{L}_{2}(\Omega)\right) \\
& \text { and a.e. in } \Omega^{T} \text {, } \\
& \chi_{n} \rightarrow \bar{\chi} \quad \text { strongly in } C\left([0, T] ; H^{1}(\Omega)\right) \\
& \text { and a.e. in } \Omega^{T} \text {, } \\
& \chi_{n, t} \rightarrow \bar{\chi}_{t} \quad \text { strongly in } L_{2}\left(0, T ; H^{1}(\Omega)\right) \cap C\left([0, T] ;\left(H^{1}(\Omega)\right)^{\prime}\right) \\
& \text { and a.e. in } \Omega^{T} \text {, }
\end{aligned}
$$




$$
\mu_{n} \rightarrow \bar{\mu} \quad \text { strongly in } C\left([0, T] ; H^{1}(\Omega)\right) \text { and a.e. in } \Omega^{T} .
$$

Moreover, due to the bounds on the dissipative terms (see (2.6))

$$
\left\|\boldsymbol{u}_{t}\right\|_{L_{2}\left(0, \infty ; \boldsymbol{H}_{0}^{1}(\Omega)\right)}+\|\nabla \mu\|_{L_{2}\left(0, \infty ; \boldsymbol{L}_{2}(\Omega)\right)} \leq c_{0},
$$

we deduce that as $n \rightarrow \infty\left(t_{n} \rightarrow \infty\right)$

$$
\boldsymbol{u}_{n, t}(\cdot)=\boldsymbol{u}_{, t}\left(t_{n}+\cdot\right) \rightarrow \mathbf{0} \quad \text { strongly in } L_{2}\left(0, \infty ; \boldsymbol{H}_{0}^{1}(\Omega)\right)
$$

and

$$
\nabla \mu_{n}(\cdot)=\nabla \mu\left(t_{n}+\cdot\right) \rightarrow \mathbf{0} \quad \text { strongly in } L_{2}\left(0, \infty ; \boldsymbol{L}_{2}(\Omega)\right) .
$$

Hence, in view of (4.15), (4.17),

$$
\overline{\boldsymbol{u}}_{t}=\mathbf{0} \quad \text { and } \quad \nabla \bar{\mu}=\mathbf{0} .
$$

This implies that $\overline{\boldsymbol{u}}$ does not depend on time and $\bar{\mu}$ does not depend on the space variables. Consequently, by (4.15),

$$
\begin{array}{ll}
\boldsymbol{u}_{n} \rightarrow \overline{\boldsymbol{u}}=\overline{\boldsymbol{u}}(0) & \text { strongly in } C\left([0, T] ; \boldsymbol{H}_{0}^{1}(\Omega)\right), \\
\boldsymbol{u}_{n, t} \rightarrow \overline{\boldsymbol{u}}_{t}=\mathbf{0} & \text { strongly in } C\left([0, T] ; \boldsymbol{H}_{0}^{1}(\Omega)\right), \\
\boldsymbol{u}_{n, t t} \rightarrow \overline{\boldsymbol{u}}_{t t}=\mathbf{0} & \text { strongly in } C\left([0, T] ; \boldsymbol{L}_{2}(\Omega)\right),
\end{array}
$$

and by $(4.14)_{1}$,

$$
\Delta \mu_{n} \rightarrow \Delta \bar{\mu}=0 \quad \text { weakly* in } L_{\infty}\left(0, T ; L_{2}(\Omega)\right) .
$$

Since $\chi_{n, t}=\Delta \mu_{n}$ in $\Omega^{T},(4.16)_{2}$ and (4.21) imply that

$$
\chi_{n, t} \rightarrow \bar{\chi}_{t}=\Delta \bar{\mu}=0 \quad \text { strongly in } C\left([0, T] ;\left(H^{1}(\Omega)\right)^{\prime}\right) .
$$

Hence, $\bar{\chi}$ does not depend on time, and in accordance with $(4.16)_{1}$,

$$
\chi_{n} \rightarrow \bar{\chi}=\bar{\chi}(0) \quad \text { strongly in } C\left([0, T] ; H^{1}(\Omega)\right) .
$$

Now, owing to (4.20)-(4.23) and recalling assumption (4.1), we deduce that

$$
\begin{aligned}
& \overline{\boldsymbol{u}}(t)=\overline{\boldsymbol{u}}(0)=\lim _{n \rightarrow \infty} \boldsymbol{u}_{n}(0)=\lim _{n \rightarrow \infty} \boldsymbol{u}\left(t_{n}\right)=\boldsymbol{u}_{\infty}, \\
& \mathbf{0}=\overline{\boldsymbol{u}}_{t}(t)=\overline{\boldsymbol{u}}_{t}(0)=\lim _{n \rightarrow \infty} \boldsymbol{u}_{n, t}(0)=\lim _{n \rightarrow \infty} \boldsymbol{u}_{t}\left(t_{n}\right)=\boldsymbol{u}_{\infty, t}, \\
& \mathbf{0}=\overline{\boldsymbol{u}}_{t t}(t)=\overline{\boldsymbol{u}}_{t t}(0)=\lim _{n \rightarrow \infty} \boldsymbol{u}_{n, t t}(0)=\lim _{n \rightarrow \infty} \boldsymbol{u}_{t t}\left(t_{n}\right)=\boldsymbol{u}_{\infty, t t},
\end{aligned}
$$

and

$$
\begin{aligned}
& \bar{\chi}(t)=\bar{\chi}(0)=\lim _{n \rightarrow \infty} \chi_{n}(0)=\lim _{n \rightarrow \infty} \chi\left(t_{n}\right)=\chi_{\infty}, \\
& 0=\bar{\chi}_{t}(t)=\bar{\chi}_{t}(0)=\lim _{n \rightarrow \infty} \chi_{n, t}(0)=\lim _{n \rightarrow \infty} \chi_{t}\left(t_{n}\right)=\chi_{\infty, t} .
\end{aligned}
$$

This proves that any element $\zeta_{\infty}=\left(\boldsymbol{u}_{\infty}, \boldsymbol{u}_{\infty, t}, \boldsymbol{u}_{\infty, t t}, \chi_{\infty}, \chi_{\infty, t}\right)$ in $\omega\left(\boldsymbol{u}_{0}, \boldsymbol{u}_{1}\right.$, $\left.\boldsymbol{u}_{2}, \chi_{0}, \chi_{1}\right)$ satisfies $(2.15)$. 
It remains to prove (2.16), (2.17). To this end we pass to the limit $n \rightarrow \infty$ in the weak formulation of (4.3)-(4.5):

$$
\begin{aligned}
& \int_{0}^{T}\left(\boldsymbol{u}_{n, t t}-\boldsymbol{Q} \boldsymbol{u}_{n}-\nu \boldsymbol{Q} \boldsymbol{u}_{n, t}, \boldsymbol{\eta}\right) d t=\int_{0}^{T}\left(z^{\prime}\left(\chi_{n}\right) \boldsymbol{B} \nabla \chi_{n}+\boldsymbol{b}_{n}, \boldsymbol{\eta}\right) d t \\
& \quad \forall \boldsymbol{\eta} \in L_{2}\left(0, T ; \boldsymbol{L}_{2}(\Omega)\right), \\
& \begin{array}{l}
\int_{0}^{T}\left(\chi_{n, t}, \xi\right) d t=\int_{0}^{T}\left(\Delta \mu_{n}, \xi\right) d t \quad \forall \xi \in L_{2}\left(0, T ; L_{2}(\Omega)\right), \\
\int_{0}^{T}\left(\mu_{n}, \varsigma\right) d t=\int_{0}^{T}\left(-\gamma \Delta \chi_{n}+\psi^{\prime}\left(\chi_{n}\right)+z^{\prime}\left(\chi_{n}\right)\right) \\
\cdot\left(\boldsymbol{B} \cdot \boldsymbol{\varepsilon}\left(\boldsymbol{u}_{n}\right)+D z\left(\chi_{n}\right)+E, \varsigma\right) d t \quad \forall \varsigma \in L_{2}\left(0, T ; L_{2}(\Omega)\right)
\end{array}
\end{aligned}
$$

where $(\cdot, \cdot)$ denotes the scalar product in $L_{2}(\Omega)$.

Clearly, by the weak convergences (4.12)-(4.14) the linear terms in (4.26) converge to the corresponding limits. The convergence of the nonlinear terms can be deduced with the help of the standard nonlinear convergence lemma (see [4, Chapter 1, Lemma 1.3]).

In fact, recalling assumptions (A3), (A4) on $z(\cdot)$ and $\psi(\cdot)$ and using estimates (4.6) we have

$$
\begin{aligned}
&\left\|\psi^{\prime}\left(\chi_{n}\right)\right\|_{L_{\infty}\left(0, T ; L_{2}(\Omega)\right)} \leq c\left(\left\|\chi_{n}\right\|_{L_{\infty}\left(0, T ; L_{6}(\Omega)\right)}^{3}+1\right) \leq c\left(c_{0}\right), \\
&\left\|z^{\prime}\left(\chi_{n}\right) \boldsymbol{B} \nabla \chi_{n}\right\|_{L_{\infty}\left(0, T ; \boldsymbol{L}_{2}(\Omega)\right)} \leq c\left\|\nabla \chi_{n}\right\|_{L_{\infty}\left(0, T ; \boldsymbol{L}_{2}(\Omega)\right)} \leq c\left(c_{0}\right), \\
& \| z^{\prime}\left(\chi_{n}\right)\left(\boldsymbol{B} \cdot \boldsymbol{\varepsilon}\left(\boldsymbol{u}_{n}\right)+D z\left(\chi_{n}\right)\right.+E) \|_{L_{\infty}\left(0, T ; L_{2}(\Omega)\right)} \\
& \leq c\left(\left\|\boldsymbol{\varepsilon}\left(\boldsymbol{u}_{n}\right)\right\|_{L_{\infty}\left(0, T ; \boldsymbol{L}_{2}(\Omega)\right)}+1\right) \leq c\left(c_{0}\right) .
\end{aligned}
$$

Thanks to these uniform estimates and the pointwise convergences (see (4.15), (4.16), (4.24), (4.25))

$$
\boldsymbol{u}_{n} \rightarrow \overline{\boldsymbol{u}}=\boldsymbol{u}_{\infty}, \quad \chi_{n} \rightarrow \bar{\chi}=\chi_{\infty} \quad \text { a.e. in } \Omega^{T},
$$

the nonlinear convergence lemma implies that

$$
\begin{aligned}
& \psi^{\prime}\left(\chi_{n}\right)=\chi_{n}^{3}-\chi_{n} \rightarrow \chi_{\infty}^{3}-\chi_{\infty}=\psi^{\prime}\left(\chi_{\infty}\right) \\
& \text { weakly* in } L_{\infty}\left(0, T ; L_{2}(\Omega)\right) \text {, } \\
& z^{\prime}\left(\chi_{n}\right) \boldsymbol{B} \nabla \chi_{n} \rightarrow z^{\prime}\left(\chi_{\infty}\right) \boldsymbol{B} \nabla \chi_{\infty} \quad \text { weakly* in } L_{\infty}\left(0, T ; \boldsymbol{L}_{2}(\Omega)\right) \text {, } \\
& z^{\prime}\left(\chi_{n}\right)\left(\boldsymbol{B} \cdot \boldsymbol{\varepsilon}\left(\boldsymbol{u}_{n}\right)+D z\left(\chi_{n}\right)+E\right) \\
& \rightarrow z^{\prime}\left(\chi_{\infty}\right)\left(\boldsymbol{B} \cdot \varepsilon\left(\boldsymbol{u}_{\infty}\right)+D z\left(\chi_{\infty}\right)+E\right) \\
& \text { weakly* in } L_{\infty}\left(0, T ; L_{2}(\Omega)\right) \text {. }
\end{aligned}
$$

Moreover, since by assumption $(\mathrm{A} 7), \boldsymbol{b} \in L_{1}\left(\mathbb{R}_{+} ; \boldsymbol{L}_{2}(\Omega)\right)$, we have

$$
\boldsymbol{b}_{n}(\cdot)=\boldsymbol{b}\left(t_{n}+\cdot\right) \rightarrow \mathbf{0} \quad \text { strongly in } L_{1}\left(0, \infty ; \boldsymbol{L}_{2}(\Omega)\right) .
$$

Consequently, passing to the limit $n \rightarrow \infty$ in (4.26) yields 


$$
\begin{gathered}
-\int_{0}^{T}\left(\boldsymbol{Q} \boldsymbol{u}_{\infty}, \boldsymbol{\eta}\right) d t=\int_{0}^{T}\left(z^{\prime}\left(\chi_{\infty}\right) \boldsymbol{B} \nabla \chi_{\infty}, \boldsymbol{\eta}\right) d t \quad \forall \boldsymbol{\eta} \in L_{2}\left(0, T ; \boldsymbol{L}_{2}(\Omega)\right), \\
\int_{0}^{T} \bar{\mu}(1, \varsigma) d t=\int_{0}^{T}\left(-\gamma \Delta \chi_{\infty}+\psi^{\prime}\left(\chi_{\infty}\right)+z^{\prime}\left(\chi_{\infty}\right)\right. \\
\left.\cdot\left(\boldsymbol{B} \cdot \boldsymbol{\varepsilon}\left(\boldsymbol{u}_{\infty}\right)+D z\left(\chi_{\infty}\right)+E\right), \varsigma\right) d t \quad \forall \varsigma \in L_{2}\left(0, T ; L_{2}(\Omega)\right) .
\end{gathered}
$$

Since $\boldsymbol{u}_{\infty}$ and $\chi_{\infty}$ do not depend on time, it follows from $(4.29)_{2}$ that $\bar{\mu}$ does not depend on time either, thus $\bar{\mu}=$ const. Moreover, the above identities reduce to

$$
\begin{gathered}
-\left(\boldsymbol{Q} \boldsymbol{u}_{\infty}, \overline{\boldsymbol{\eta}}\right)=\left(z^{\prime}\left(\chi_{\infty}\right) \boldsymbol{B} \nabla \chi_{\infty}, \overline{\boldsymbol{\eta}}\right) \quad \forall \overline{\boldsymbol{\eta}} \in \boldsymbol{L}_{2}(\Omega), \\
(\bar{\mu}, \bar{\varsigma})=\left(-\gamma \Delta \chi_{\infty}+\psi^{\prime}\left(\chi_{\infty}\right)+z^{\prime}\left(\chi_{\infty}\right)\left(\boldsymbol{B} \cdot \boldsymbol{\varepsilon}\left(\boldsymbol{u}_{\infty}\right)\right.\right. \\
\left.\left.+D z\left(\chi_{\infty}\right)+E\right), \varsigma\right) \quad \forall \bar{\varsigma} \in L_{2}(\Omega) .
\end{gathered}
$$

Hence, recalling that (see (4.11), (4.24), (4.25)) $\boldsymbol{u}_{\infty} \in \boldsymbol{H}^{2}(\Omega) \cap \boldsymbol{H}_{0}^{1}(\Omega)$, $\chi_{\infty} \in H_{N}^{2}(\Omega)$, it follows that $\boldsymbol{u}_{\infty}, \chi_{\infty}$ satisfy the system

$$
\begin{array}{cc}
-\boldsymbol{Q} \boldsymbol{u}_{\infty}=z^{\prime}\left(\chi_{\infty}\right) \boldsymbol{B} \nabla \chi_{\infty} & \text { in } \Omega, \\
\boldsymbol{u}_{\infty}=\mathbf{0} & \text { on } S, \\
-\gamma \Delta \chi_{\infty}+\psi^{\prime}\left(\chi_{\infty}\right) & \\
+z^{\prime}\left(\chi_{\infty}\right)\left(\boldsymbol{B} \cdot \boldsymbol{\varepsilon}\left(\boldsymbol{u}_{\infty}\right)+D z\left(\chi_{\infty}\right)+E\right)=\bar{\mu} & \text { in } \Omega, \\
\boldsymbol{n} \cdot \nabla \chi_{\infty}=0 & \text { on } S, \\
f_{\Omega} \chi_{\infty} d x=\chi_{m}, &
\end{array}
$$

where $\bar{\mu}$ is a constant.

Clearly, in view of (1.11) the above system is equivalent to (2.16), (2.17). Thus the proof of Theorem 2.2 is complete.

\section{References}

[1] E. Bonetti, Modelling phase transitions via entropy equation: Long-time behaviour of the solutions, in: Dissipative Phase Transitions, P. Colli et al. (eds.), Ser. Adv. Math. Appl. Sci. 71, World Sci., 2006, 21-42.

[2] E. Bonetti and E. Rocca, Global existence and long-time behaviour for a singular integro-differential phase-field system, Comm. Pure Appl. Anal. 6 (2007), 367-387.

[3] C. M. Dafermos, Asymptotic behavior of solutions of evolution equations, in: Nonlinear Evolution Equations, M. Crandall (ed.), Academic Press, New York, 1978, 103-123.

[4] J.-L. Lions, Quelques méthodes de résolution des problèmes aux limites non linéaires, Dunod, Paris, 1969.

[5] I. Pawłow and W. M. Zajączkowski, Global regular solutions to Cahn-Hilliard system coupled with viscoelasticity, Math. Methods Appl. Sci. 32 (2009), 2197-2242. 
[6] J. Simon, Compact sets in the space $L^{p}(0, T ; B)$, Ann. Mat. Pura Appl. 146 (1987), 65-97.

[7] S. Zheng, Asymptotic behavior of solution to the Cahn-Hilliard equation, Appl. Anal., 23 (1986), 165-184.

Irena Pawłow

Systems Research Institute

Polish Academy of Sciences

Newelska 6

01-447 Warszawa, Poland

E-mail: pawlow@ibspan.waw.pl

and

Institute of Mathematics and Cryptology

Cybernetics Faculty

Military University of Technology

Kaliskiego 2

00-908 Warszawa, Poland
Wojciech M. Zajączkowski

Institute of Mathematics

Polish Academy of Sciences

Śniadeckich 8

00-956 Warszawa, Poland

E-mail:wz@impan.pl

$$
\text { and }
$$

Institute of Mathematics and Cryptology

Cybernetics Faculty

Military University of Technology

Kaliskiego 2

00-908 Warszawa, Poland

Received 18.12.2008

and in final form 9.6.2009 
